\title{
Outcome of Chondroblastoma Treated With Intralesional Curettage and Autogenous Iliac Bone Graft: A Retrospective Study
}

\section{Lei Yang}

Sichuan University West China Hospital

\section{Panyi Yang}

Sichuan University West China Hospital

Daoxi Wang

Sichuan University West China Hospital

Xiaodong Yang

Sichuan University West China Hospital

Lang Li

Sichuan University West China Hospital

xueyang Tang ( $\nabla$ xueyangtwch@163.com )

Sichuan University West China Hospital

\section{Research}

Keywords: Chondroblastoma, child, prognosis, bone tumor

Posted Date: October 9th, 2020

DOI: https://doi.org/10.21203/rs.3.rs-88880/v1

License: (c) (1) This work is licensed under a Creative Commons Attribution 4.0 International License. Read Full License 


\section{Abstract}

\section{Background}

Chondroblastoma is a rare benign tumor mainly occurred in epiphyses and apophyses of long bones, and usually affects the young people. The primary treatment for chondroblastoma is intralesional curettage. The aim of this study was to evaluate the clinical result of patients suffering chondroblastoma treated with intralesional curettage, electrocauterization of the cavity and autogenous iliac bone grafting.

\section{Methods}

From January 2000 to January 2010, 15 patients with chondroblastoma were treated with intralesional curettage and electrocauterization of the cavity, and 14 of them received autogenous iliac bone grafting. After operation, patients were immobilized with splint for 4 weeks and followed up for at least 10 years. The recurrence was examined by clinical manifestation and $X$ ray. The clinical outcome included complications and the Musculoskeletal Tumor Society Score (MSTS).

\section{Results}

Totally, 15 patients with chondroblastoma were enrolled in our study, including 9 males and 6 females, with a mean age of 13 years old (range from 8 to 21 years). The most common chief compliant were pain and limited joint motion, others including local swelling and increased skin temperature. The mean duration of symptom was 5 months (range from 2-14 months). Tumors mostly affected the proximal humerus (7/15), distal femur (4/15), proximal femur (1/15), proximal tibia (2/15) and femoral head (1/15). With a mean follow up of 12years (range from 10-16 years) , two patients had complications of upper limb shortening and one patient suffered re-operation due to tumor recurrence. The mean postoperative MSTS was 28.1 (range, 24 to 30 ) points, which was significantly higher than the preoperative score of 17.6 (range, 12 to 21 ) points.

\section{Conclusions}

Thorough curettage with electrocauterization and autogenous iliac bone grafting has good functional outcomes in the treatment of chondroblastoma, but still has a low risk of recurrence and complications.

Level of Evidence

Level IV, retrospective case series.

\section{Background}

Chondroblastoma is a rare benign aggressive tumor mainly occurred in epiphyses and apophyses of long bones, which usually affects the young people[1]. The proximal humerus, proximal tibia and distal femur are the most common sites involved[2]. Clinical manifestations of the disease are not typical, including 
pain which is the most common presentation and even sometimes may be the only one, local swelling, limited motion of the adjacent joint, and joint effusion[3].

The primary treatment for chondroblastoma is intralesional curettage with bone grafting in a large defect[4], and some authors preferred chemical or physical adjuvants inactivation to decrease tumor recurrence[5]. The local recurrence rates were obviously different in the literature, which could be as high as $30 \%[6,7]$.

The aim of this study was to retrospectively evaluate the clinical outcome of patients suffering chondroblastoma treated with intralesional curettage, electrocauterization of the cavity and autogenous iliac bone grafting from January 2000 to January 2010 in our center. The details of the patients' general data and clinical results are presented here.

\section{Patients And Methods}

This study was approved by the Ethic Committee on Biomedical Research, West China Hospital of Sichuan University in accordance with the Declaration of Helsinki. Written informed consent was obtained from the patients for the publication of this report and any accompanying images.

All 15 patients received open surgery for intralesional curettage, electrocauterization of the lesion cavity and the final pathological diagnosis. Autogenous iliac bone grafting were performed in 14 patients, and 2 of them received autogenous bone grafting with artificial bone due to the large defect. One patient suffering femoral head chondroblastoma was only treated with intralesional curettage, electrocauterization of the cavity, without grafting. Surgery was performed under general anesthesia by a senior orthopedic surgeon who was well trained. Postoperatively, the extremity was placed in a wellpadded splint $t$ for 4 weeks with no weight bearing. All patients were followed up at least for 10 years, with a mean follow up of 12years (range from 10-16 years). Patients were followed up every two months throughout the first one year, then every six months in the second year. After the second year, patients were followed up every 2 years. Local recurrences and lung metastasis were examined by clinical manifestation and $X$ ray. The clinical outcome included complications, limb length and Musculoskeletal Tumor Society Score (MSTS).

\section{Results}

Totally 15 patients (9 male and 6 female) were enrolled in our study, with a mean age of 13 years old (range from 8 to 21 years). The major clinical manifestations included 15 cases of pain, 5 cases of local swelling, 7 cases of limited movement of adjacent joints and 1 case of increased skin temperature. The mean duration of symptom was 5 months (range from 2-14 months).

Only 7 patients were considered a possibility of chondroblastoma preoperative. Eight patients were undiagnosed or misdiagnosed before operation, including 1 case of bone cyst, 1 case of bone tuberculosis, 1 case of osteofibrous dysplasia, and 5 cases of undiagnosed bone tumors. 
In this group of patients, chondroblastoma mainly affected the humerus (Fig.1) and femur in a single site. There was 1 patient with 2 lesions involved the proximal tibia and distal femur of the left side who was considered a possibility of osteofibrous dysplasia preoperatively (Fig.2). The lesions located in proximal humerus, distal femur, proximal femur, proximal tibia and femoral head were 7, 4, 1, 2 and 1 , respectively.

Among these 15 patients, 11 patients had persistent epiphysis, 3 of them with lesions extended through the epiphyseal plate and 8 patients with lesions located inside the epiphysis. Focal articular surface destruction of the femoral head was involved in 1 patient (Fig.3), who received intralesional curettage through a hip joint approach and electrocauterization of lesion cavity without bone grafting, and gained a normal hip function with no tumour recurrence, no femoral head necrosis or arthritis during 10 years follow up.

Postoperatively, one patient developed a surgical wound infection with a delay of incision healing after treatment with antibiotics and subcutaneous drainage.During the follow up, two patients had complications of limb shortening that were within $2 \mathrm{~cm}$ and both involved the upper limbs.

During follow up, all patients had good clinical outcomes, with a postoperative MSTS score of 28.1 (range, 24 to 30) points, which was significantly higher than the preoperative score of 17.6 (range, 12 to 21) points. While 1 patient developed local recurrence in the proximal humerus 9 months after the initial treatment of intralesional curettage, electrocauterization of the cavity and autogenous iliac bone graft. This patient received a re-operation of extended lesion curettage with bone cement filling and no recurrence was observed. Lung metastasis was not observed in this group of patients.

\section{Discussion}

Chondroblastoma is a rare benign bone tumor that typically arise during the teenage years with a wide range of age[8]. It mostly affects the epiphyses or apophyses of long bones such as humerus, femur and tibia. Less common locations including patella, calcaneus, acetabulum and spine, also had been reported in literature[9-11].It seems that chondroblastoma occurs more frequently in males than in females, with a ratio of 2.3 to 1 in literature[2,12]. In our study of chondroblastoma, the mean age of onset was 13 years old with a male to female ratio of 1.5:1, and also the proximal humerus was the mostly involved location, which all coincided with most previous studies.

A characteristic imaging finding may be very helpful for diagnosis, even though the clinical manifestation of chondroblastoma is not typical and the preoperative diagnosis is difficult[4]. The typical imaging features include a eccentric round-like focus located in epiphysis with a lytic lesion, calcification or a peripheral sclerotic margin, and usually a significantly higher-intensity signal on T2-weighted MRI[13,14]. El-Ali AM et al[15] reported that chondroblastoma was one of the most common disease presented as solitary long-bone epiphyseal lesions, and characterized as peripherally location, discrete T1-weighted hypointense rim, larger in patient age and lesion size when compared to the less common osteomyelitis. Blancas $C$ et al[16] also found these common imaging appearances as well as periosteal reaction and 
perilesional edema. Patients with these typical imaging features, lesion locations and the appropriate age group should be highly in consideration of a presumptive diagnosis of chondroblastoma, even though biopsy and pathologic diagnosis may still be needed[17].

Surgical removal of the lesion and bone grafting in a large defect is the standard treatment for chondroblastoma[1,2]. For completely curettage of tumor and avoid recurrence, most authors advised adjuvant physical or chemical inactivation methods, including high-speed burr, electrocauterization, phenol, cryotherapy and ethanol[5,18,19]. Ebeid WA et al[4] found a significantly lower recurrence rate in patients treated with thorough curettage and high-speed burr. Zekry KM et al[20] reported that extended curettage using high-speed burr with phenol and ethanol as local adjuvants was effective in lowering the incidence of local recurrence. However, there is still no study on the comparison of these different adjuvant options and point out the optimal choice. Moreover, adjuvant therapies may cause an extensive tissue damage while killing tumor cells, and should be used carefully, especially for tumors involving the epiphyseal plate[5].

Due to the typical chondroblastoma location in epiphysis, there was always a concern about the physeal plate injury of patients with persistent epiphysis, which might cause growth disturbance[6]. While, it seems that a completely extended lesions curettage is more important than protection of the epiphyseal plate to avoid local recurrence[20]. Mashhour and Abdel Rahman[21] preferred aggressive surgical treatment at the time of the first surgery to prevent recurrence, because re-operation after recurrence might cause more damage on growth plate. Liu Q et al[22] also reported that intraregional aggressive curettage, phenolization, and allogeneic bone grafting were effective for treatment of chondroblastoma with low recurrence. However, lesions extended through the epiphyseal plate or an aggressive curettage does lead to epiphyseal plate injury. Xiong $Y$ et al [23] found that the lesion crossing the epiphyseal line more led to more severe limb length discrepancy, while all 5 patients with upper limb shorting in their study had satisfied shoulder function. In this group of patients, complications of upper limb shortening occurred on 2 patients who both had persistent epiphysis with lesions extended through the epiphyseal plate. Because the shorting were within $2 \mathrm{~cm}$ and occurred on upper limbs with no obvious effects on function or appearance, no further treatment was carried out.

Cortical defect and fracture had been reported before when chondroblastoma affected the articular surface,and there might be a complication of arthritis in long term follow up[24]. In a retrospective study focused on the joint status by Farfalli GL et al [25], osteoarthritis was the most common complication of aggressive curettage of epiphyseal chondroblastoma, which contributed to $77 \%$ of the overall complications and four of these patients with complication of osteoarthritis finally received joint replacement. In this study, focal articular surface destruction of the femoral head was involved in one patient who gained a good functional outcome without osteoarthritis complication after treatment of intralesional curettage and electrocauterization. Treatment of femoral head chondroblastoma can be extremely challenging. Surgical approaches include direct hip joint approach and indirect way from the lateral femur to femur head via a subtrochanteric drill hole. Laitinen MK[2] preferred the direct hip joint approach to treat femur head lesion without grafting. In their study, results of femoral head 
chondroblastoma treated in a direct hip joint approach were better with no local recurrence or femoral head collapse occurred, when compared to the indirect surgical approach. Liu Q et al [26]reported a modified trapdoor procedures and surgical dislocation as a safe and effective techniques for treating chondroblastoma in the femoral head, while osteonecrosis of femoral head or secondary osteoarthritis still could not be avoided.

The prognosis of chondroblastoma generally is good, while it is still an aggressive tumor, with risk of local recurrence and extremely rare metastasis[27,28]. Location, age, persist epiphysis and inadequate curettage once were considered a possible risk factor for recurrence, but still lack of reliable evidence or controversial[$[7,13,24]$. Outani $\mathrm{H}$ et al[30] reported a $20 \%$ recurrence rate in their retrospective study of 40 patients with chondroblastoma, while the univariate analysis did not identify any significant risk factors for local recurrence. Ebeid WA et al [4] reported a retrospective study of 91 patients with chondroblastoma and found no statistical significance between the incidence of local recurrence and factors including age, sex, pathologic fracture or even the type of filler used. Nevertheless, most authors still believed that inadequate curettage was the most significant risk factor associated with local recurrence[2,6,29]

The limitations of our study were that it was a retrospective study in a single center, and it was in a small sample size with no control. There still need more further studies of large sample, muti-centre clinical trials, especially in the adjuvant treatment and risk factors of local recurrence.

\section{Conclusions}

Thorough intralesional curettage with electrocauterization of the cavity and bone grafting is an effective treatment for chondroblastoma. Although most patients will gain good clinical outcome, there are still a few patients at risk of recurrence and complications.

\section{Abbreviations}

MSTS: Musculoskeletal Tumor Society Score;

CT: computed tomography;

MRI: magnetic resonance imaging

\section{Declarations}

\section{Ethics approval and Consent to participate}

This study was approved by the Ethic Committee on Biomedical Research, West China Hospital of Sichuan University in accordance with the Declaration of Helsinki (Committee's reference number:1085). Written informed consent was obtained from all individual participants included in the study.

\section{Consent for publication}


Written informed consent for publication was obtained from all adult participants and parents of all pediatric participants.

\section{Availability of data and materials}

The datasets used during the current study are available from the corresponding author on reasonable request.

\section{Competing interests}

The authors declare that they have no competing interests.

\section{Funding}

This research did not receive any specific grant from funding agencies in the public, commercial, or notfor-profit sectors, or any pharmaceutical and industry support.

\section{Authors' contributions}

LY wrote the first draft of the manuscript, completed the analysis and interpretation of data for the work; PYY, DXW, XDY and LL completed the patients' follow up and data collection. XYT performed the clinical practice and final approval of the version to be published. All authors reviewed the manuscript for important intellectual content and approved the final version to be published.

Acknowledgements: Not Applicable.

\section{References}

1. Chen W, DiFrancesco LM. Chondroblastoma: An Update. Arch Pathol Lab Med. 2017;141(6):867-871.

2. Laitinen MK, Stevenson JD, Evans S, et al. Chondroblastoma in pelvis and extremities- a single centre study of 177 cases. J Bone Oncology. 2019;17:100248.

3. Jundt G, Baumhoer D. Chondroblastom. Pathologe. 2018;39(2):132-138.

4. Ebeid WA, Hasan BZ, Badr IT, et al. Functional and Oncological Outcome After Treatment of Chondroblastoma With Intralesional Curettage. Journal of Pediatric Orthopedic. 2019;39(4):e312e317.

5. Özer D, Arıkan Y, Gür V, et al. Chondroblastoma: An evaluation of the recurrences and functional outcomes following treatment. Acta Orthop Traumatol Turc. 2018;52(6):415-418.

6. Suneja R, Grimer RJ, Belthur M, et al. Chondroblastoma of bone: long-term results and functional outcome after intralesional curettage. J Bone Joint Surg Br. 2005;87(7):974-8

7. Atalar H, Basarir K, Yildiz Y, et al. Management of Chondroblastoma : retrospective review of 28 patients. J Orthop Sci. 2007;12(4): 334-40. 
8. Angelini A, Hassani M, Mavrogenis AF, et al. Chondroblastoma in adult age. Eur J Orthop Surg Traumatol. 2017;27(6):843-849.

9. Lang Y, Yu Q, Liu Y, et al. Chondroblastoma of the patella with pathological fracture in an adolescent: a case report. World J Surg Oncol. 2019;17(1):218.

10. Dutt L, Schade VL, Manoso MW. Calcaneal chondroblastoma with pathologic fracture and recurrence. J Foot Ankle Surg. 2015;54(2):258-267.

11. Venkatasamy A, Chenard MP, Massard G, et al. Chondroblastoma of the thoracic spine: a rare location. Case report with radiologic-pathologic correlation. Skeletal Radiol. 2017;46(3):367-372.

12. Konishi E, Nakashima Y, Mano M, et al. Chondroblastoma of extra-craniofacial bones: clinicopathological analyses of 103 cases.Pathol.Int.2017;67(10):495-502.

13. Sailhan F, Chotel F, Parot R, et al. Chondroblastoma of bone in a pediatric population. J Bone Joint Surg Am. 2009;91(9):2159-2168.

14. Edel G, Ueda Y, Nakanishi J, et al. Chondroblastoma of bone. A clinical, radiological, light and immunohistochemical study. Virchows Arch A Pathol Anat Histopathol. 1992;421(4):355-66.

15. El-Ali AM, Coblentz A, Degnan AJ. Solitary long-bone epiphyseal lesions in children: radiologicpathological correlation and epidemiology. Pediatr Radiol. 2020. doi: 10.1007/s00247-020-04752-8.

16. Blancas C, Llauger J, Palmer J, et al. Imaging findings in chondroblastoma. Radiologia. 2008;50(5):416-423.

17. Maheshwari AV, Jelinek JS, Song AJ, et al. Metaphyseal and diaphyseal chondroblastomas. Skeletal Radiol. 2011;40(12):1563-73.

18. Van Der Geest IC, Van Noort MP, Schreuder HW, et al. The cryosurgical treatment of chondroblastoma of bone: long term oncologic and functional results. J Surg Oncol. 2007;96(3):230-234.

19. Yang Z, Tao H, Ye Z, et al. The diagnosis and treatment of tibial intercondylar chondroblastoma. Clinics (Sao Paulo). 2018;73:e540.

20. Zekry KM, Yamamoto N, Hayashi K, et al. Surgical treatment of chondroblastoma using extended intralesional curettage with phenol as a local adjuvant. J Orthop Surg (Hong Kong). 2019;27(3):2309499019861031.

21. Mashhour MA, Abdel Rahman M. Lower recurrence rate in chondroblastoma using extended curettage and cryosurgery. Int Orthop (SICOT). 2014;38(5): 1019-1024.

22. Liu Q, He H, Yuan $Y$, et al. Have the difficulties and complications of surgical treatment for chondroblastoma of the adjoining knee joint been overestimated? J Bone Oncol. 2019; 13;17:100240.

23. Xiong $Y$, Lang $Y, Y u$ Z, et al. The effects of surgical treatment with chondroblastoma in children and adolescents in open epiphyseal plate of long bones. World J Surg Oncol. 2018;16(1):14.

24. Xu H, Nugent D, Monforte HL, et al. Chondroblastoma of bone in the extremities: a multicenter retrospective study. J Bone Joint Surg Am. 2015;97(11):925-931. 
25. Farfalli GL, Slullitel PA, Muscolo DL, et al. What Happens to the Articular Surface After Curettage for Epiphyseal Chondroblastoma? A Report on Functional Results, Arthritis, and Arthroplasty. Clin Orthop Relat Res. 2017;475(3):760-766.

26. Liu Q, He HB, Zeng H, et al. Modified trapdoor procedures by surgical dislocation approach to treat chondroblastoma of the femoral head. Bone Joint J. 2019;101-B(6):732-738.

27. Sohn SH, Koh SA, Kim DG, et al. A case of spine origin chondroblastoma metastasis to lung. Cancer Res Treat. 2009;41(4):241-244.

28. Focaccia M, Gambarotti M, Hakim R, et al. Chondroblastoma's Lung Metastases Treated with Denosumab in Pediatric Patient. Cancer Res Treat. 2020. doi: 10.4143/crt.2020.384.

29. Lin PP, Thenappan A, Deavers MT, et al. Treatment and prognosis of chondroblastoma. Clin Orthop Relat Res. 2005;438:103-109.

30. Outani H, Kakunaga S, Hamada K, et al. Clinical outcomes of chondroblastoma treated using synthetic bone substitute: risk factors for developing radiographic joint degeneration. World J Surg Oncol. 2020;2;18(1):47.

\section{Figures}




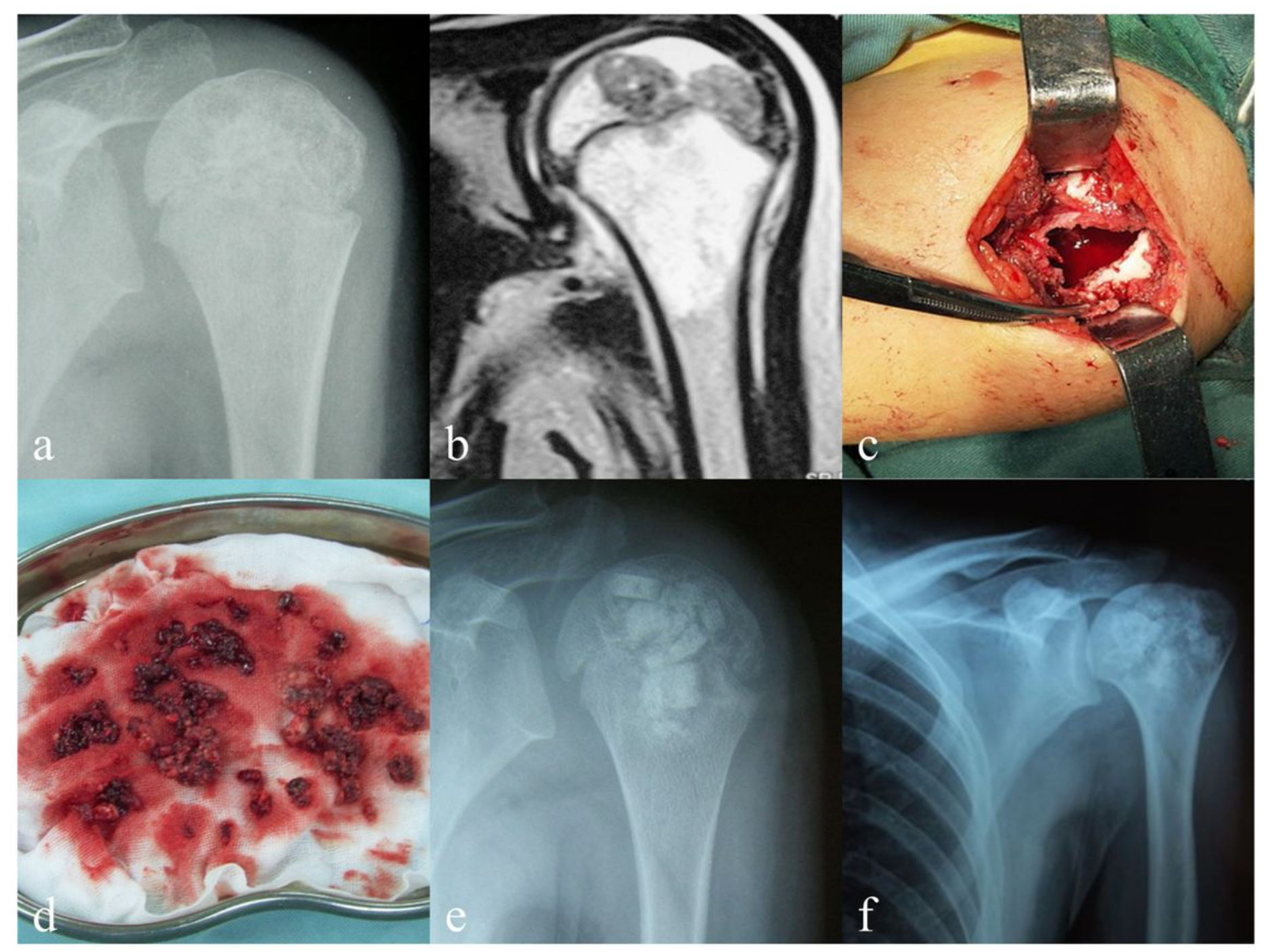

\section{Figure 1}

An 11 years old boy with chondroblastoma involved the proximal humerus of the left side, and treated with intralesional curettage, electrocauterization of the cavity and bone grafting. a,b Radiographs showing the lesions on X ray film and CT scan.c Intraoperative photo after curettage. d Histological appearance of the tumor. e Postoperative radiograph showing the lesions after curettage and grafting. $f$ Radiograph at 10 years follow up. CT: computed tomography. 


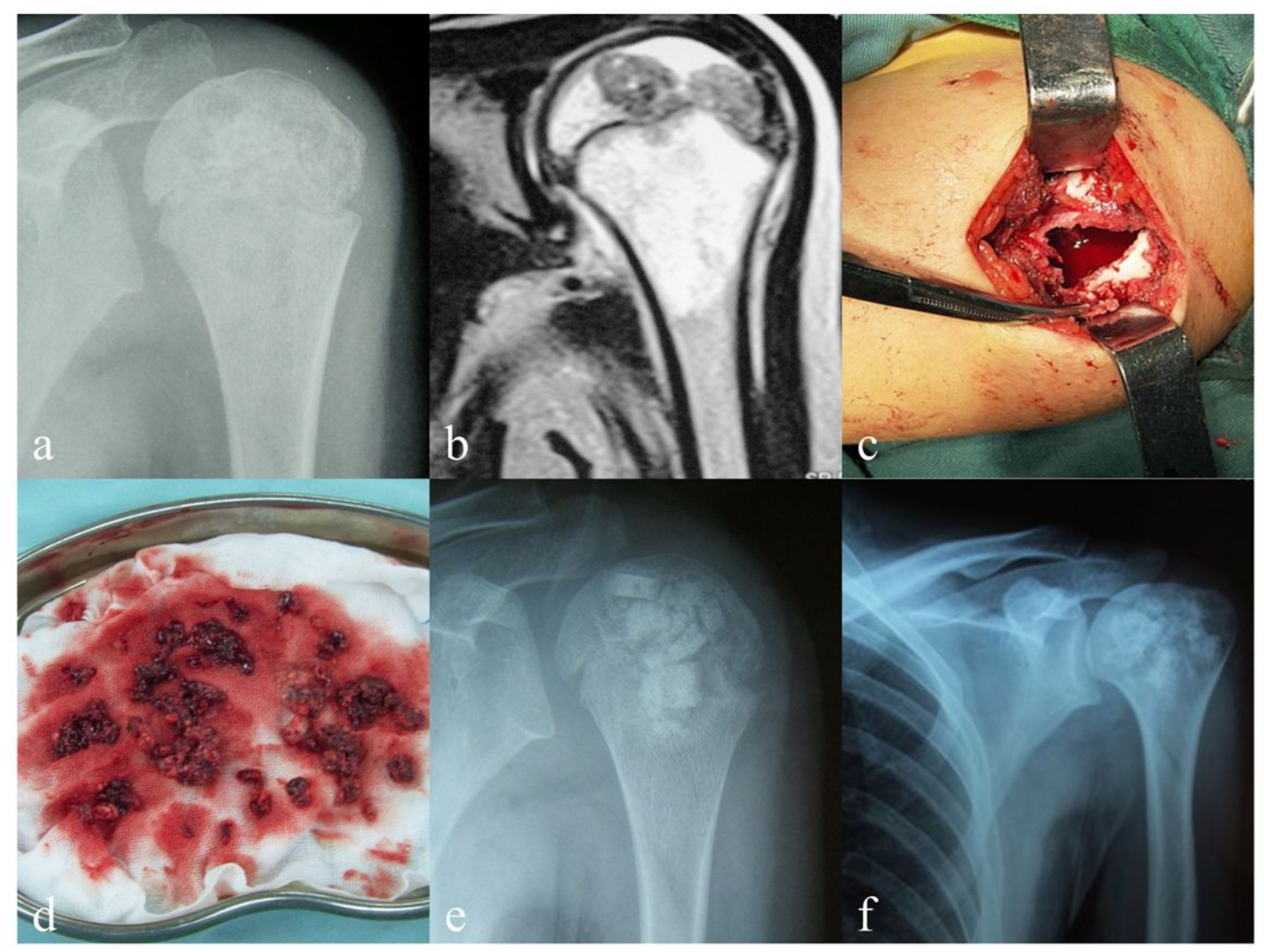

\section{Figure 1}

An 11 years old boy with chondroblastoma involved the proximal humerus of the left side, and treated with intralesional curettage, electrocauterization of the cavity and bone grafting. a,b Radiographs showing the lesions on $\mathrm{X}$ ray film and CT scan.c Intraoperative photo after curettage. $d$ Histological appearance of the tumor. e Postoperative radiograph showing the lesions after curettage and grafting. $f$ Radiograph at 10 years follow up. CT: computed tomography. 


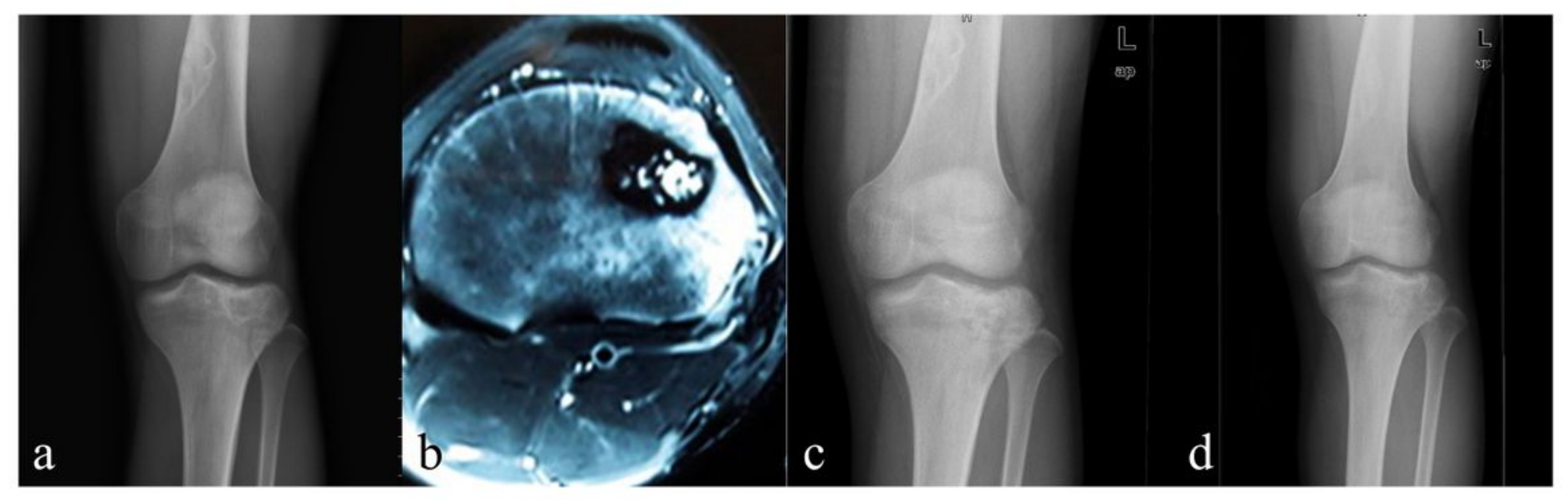

\section{Figure 2}

A 16 years old male patient with 2 lesions involved the proximal tibia and distal femur of the left side who was considered a possibility of osteofibrous dysplasia preoperatively. Operation of intralesional curettage, electrocauterization of the cavity and autogenous iliac bone grafting was only performed on the tibia due to a persistent pain of lower knee. a Anteroposterior view showing the lesions on $\mathrm{X}$ ray. $\mathrm{b}$ MRI of the lesions. c Postoperative radiograph showing the lesions after curettage and grafting. $d$ Radiograph at 10 years follow up.MRI: magnetic resonance imaging.

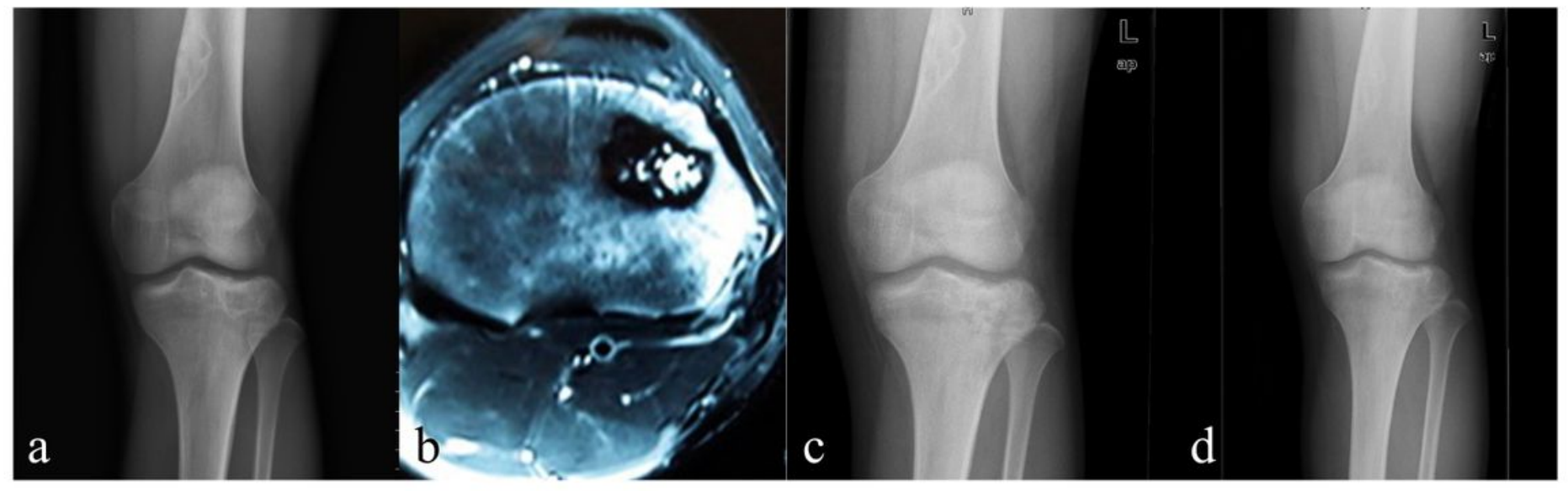

\section{Figure 2}

A 16 years old male patient with 2 lesions involved the proximal tibia and distal femur of the left side who was considered a possibility of osteofibrous dysplasia preoperatively. Operation of intralesional curettage, electrocauterization of the cavity and autogenous iliac bone grafting was only performed on the tibia due to a persistent pain of lower knee. a Anteroposterior view showing the lesions on $\mathrm{X}$ ray. $\mathrm{b}$ MRI of the lesions. c Postoperative radiograph showing the lesions after curettage and grafting. $d$ Radiograph at 10 years follow up.MRI: magnetic resonance imaging. 


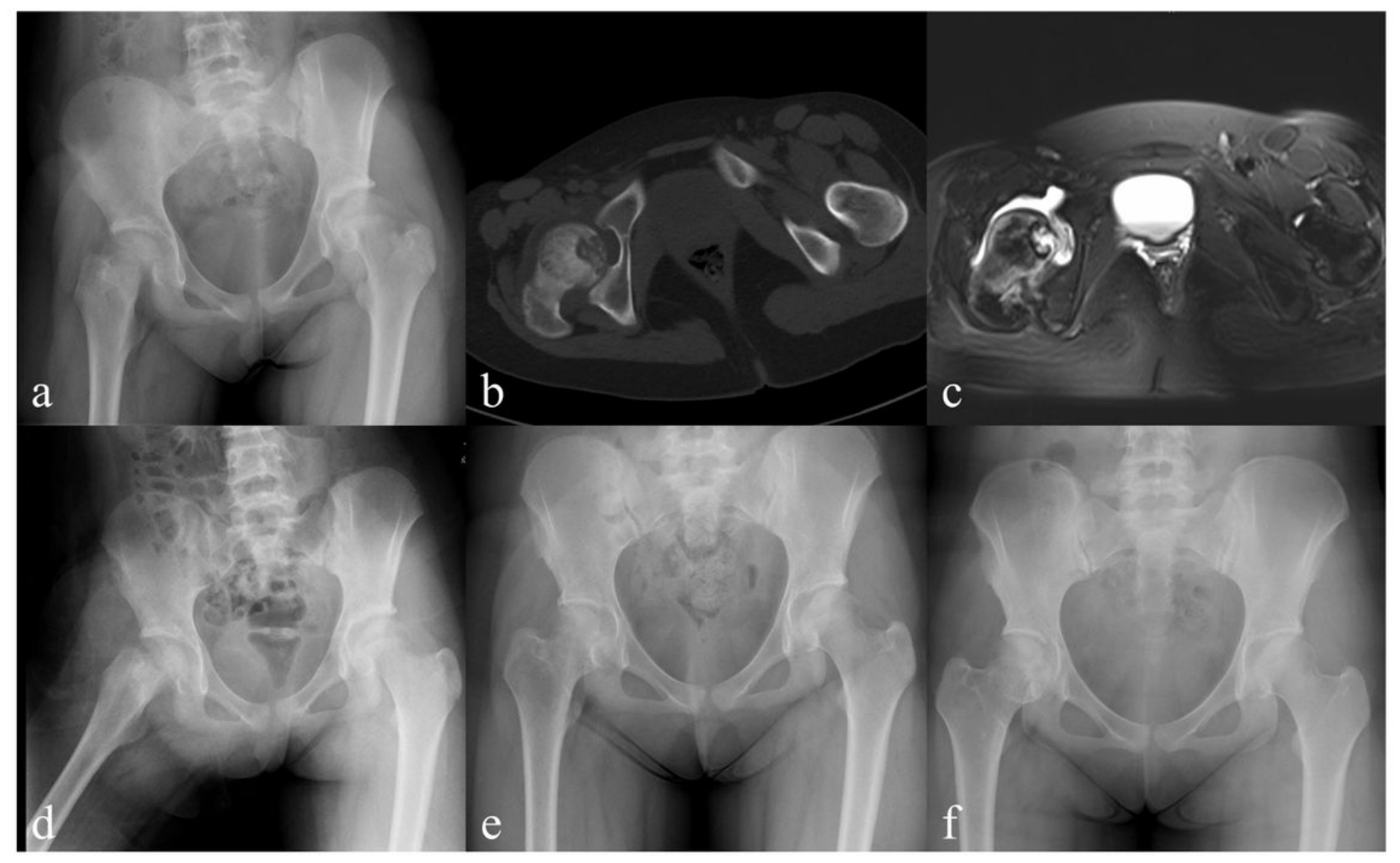

Figure 3

A 12 years old girl with chondroblastoma involved the femur head of the right side, who treated with intralesional curettage, electrocauterization of the cavity and no grafting. a Radiograph of the lesion on right femur head. b CT scan of the lesion. c MRI of the lesion. $d$ Postoperative radiograph showing the lesion after curettage. e,f Radiographs at 5 years and 10 years of follow up. CT: computed tomography; MRI: magnetic resonance imaging. 


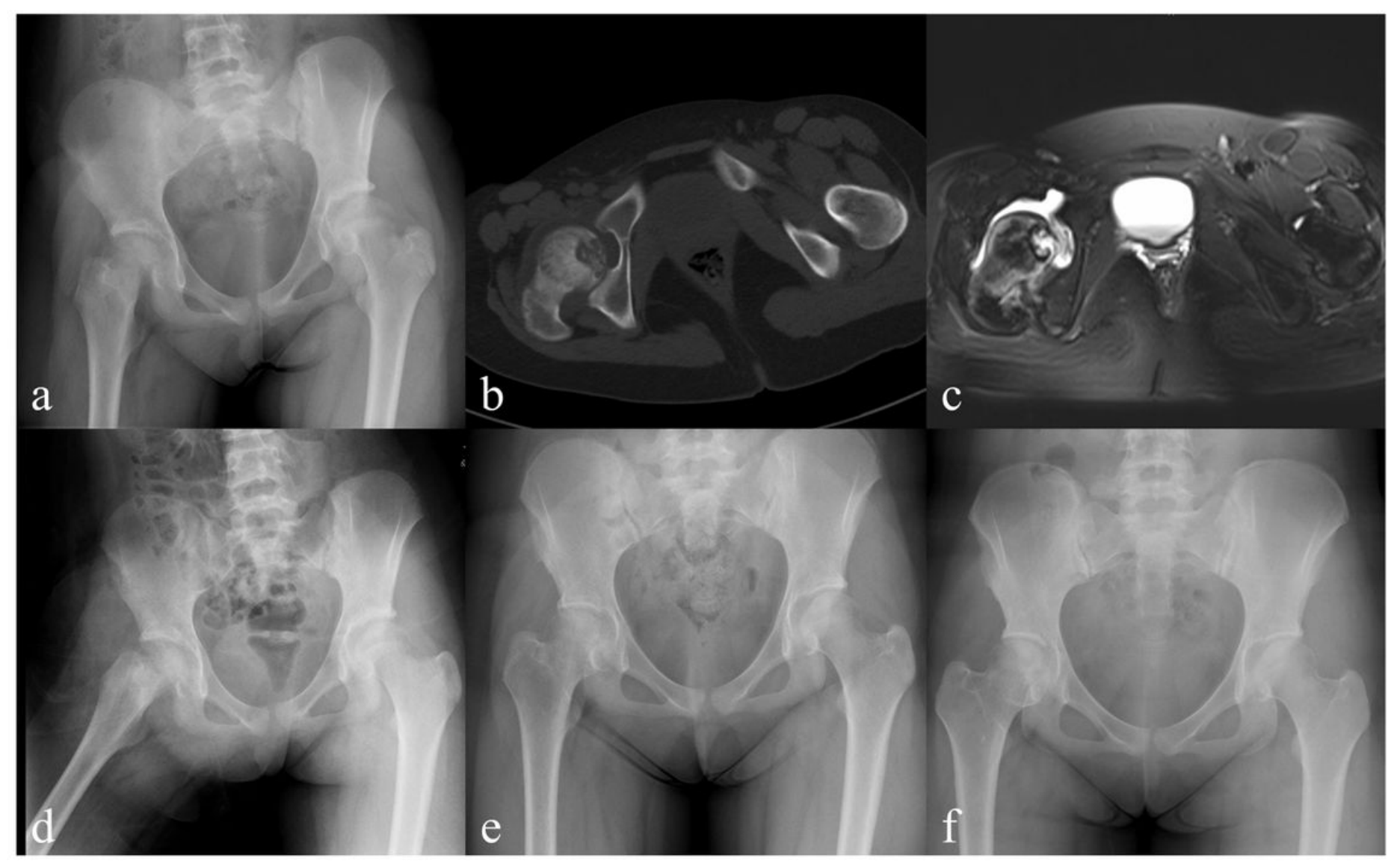

Figure 3

A 12 years old girl with chondroblastoma involved the femur head of the right side, who treated with intralesional curettage, electrocauterization of the cavity and no grafting. a Radiograph of the lesion on right femur head. b CT scan of the lesion. c MRI of the lesion. $d$ Postoperative radiograph showing the lesion after curettage. e,f Radiographs at 5 years and 10 years of follow up. CT: computed tomography; MRI: magnetic resonance imaging. 